doi:10.13108/2021-13-3-44

\title{
ON NON-LOCAL PROBLEMS FOR THIRD ORDER EQUATION WITH CAPUTO OPERATOR AND NON-LINEAR LOADED PART
}

\author{
B.I. ISLOMOV, O.KH. ABDULLAEV
}

\begin{abstract}
This paper is devoted to proving the unique solvability of nonlocal problems with an integral conjugate condition for one class of third-order equations with a parabolichyperbolic operator including the Caputo fractional derivative and a nonlinear term containing the trace of the solution $u(x, 0)$. Since the considered equation is of the third order, in which a first order differential operator with coefficients $a, b$ and $c$ acts on a parabolichyperbolic second order operator, the coefficients $a, b$ and $c$ influence essentially a welldefined formulation of boundary value problems. This is why, before providing complete formulation of the studied problems, we present the boundary conditions in their formulation for various cases of the behavior of the coefficients $a, b$ and $c$.

In the first part of the paper we formulate a nonlocal Problem I with an integral conjugate condition in the case $0<b / a \leqslant 1$. This problem is equivalently reduced to a Volterra type nonlinear integral equation and we prove its unique solvability by the successive approximations method.

The second part of the work is devoted to well-posed formulation and to studying other nonlocal problems, the formulations of which are related with other possible cases of $a$ and $b$. We provide a detailed study of Problem II. Then as remarks we described the way of studying other formulated problems.
\end{abstract}

Keywords: parabolic-hyperbolic operator, Caputo fractional derivative, nonlinear loaded term, integral conjugate condition, nonlinear integral equation.

Mathematics Subject Classification: 35M10, 34K37, 35R11

\section{INTRODUCTION}

Let $\Omega$ be a simply connected domain enveloped in the half-plane $y>0$ by the segments $B B_{0}$, $B_{0} A_{0}, A_{0} A$ located respectively on the straight lines $x=1, y=h, x=0$ and, in the half-plane $y<0$, by the characteristics

$$
\begin{aligned}
& A C: \quad x+y=0, \\
& B C: \quad x-y=1
\end{aligned}
$$

of the equation

$$
\left(a \frac{\partial}{\partial x}+b \frac{\partial}{\partial y}+c\right) L u=0
$$

B.I. Islomov, O.Kh. Abdullaev, On non-local problems for third order equation with CaPUTO OPERATOR AND NON-LINEAR LOADED PART.

(C) Islomov B.I., Abdullaev O.Kh. 2021.

Submitted July 1, 2020. 
where $C\left(\frac{1}{2},-\frac{1}{2}\right) ; a, b$ and $c$ are real constants and $a^{2}+b^{2} \neq 0$,

$$
L u \equiv \begin{cases}L_{1} u=\frac{\partial^{2} u}{\partial x^{2}}-{ }_{c} D_{0 y}^{\alpha} u+f_{1}(x, y ; u(x, 0)), & (x, y) \in \Omega_{1}, \\ L_{2} u=\frac{\partial^{2} u}{\partial x^{2}}-\frac{\partial^{2} u}{\partial y^{2}}+f_{2}(x, y ; u(x, 0)), & (x, y) \in \Omega_{2},\end{cases}
$$

${ }_{c} D_{a y}^{\alpha}$ is a Caputo differential operator of order $0<\alpha<1$ [1], [2]:

$$
{ }_{c} D_{0 y}^{\alpha} f=\frac{1}{\Gamma(1-\alpha)} \int_{0}^{y}(y-t)^{-\alpha} f^{\prime}(t) d t
$$

and

$$
\Omega_{1}=\Omega \cap\{y>0\}, \quad \Omega_{2}=\Omega \cap\{y<0\} .
$$

It is well-known that local problems for equation (1.1) with continuous and discontinuous conjugate conditions as $f_{i}(x, y ; u(x, 0))=0$ and $\alpha=1$, were studied in work [3]. For a third order equation with a parabolic-hyperbolic operator of an integer order involving linear loaded parts, only local problems were studied with continuous conjugate condition, see [4], [5]. It should be noted by the methods employed in works by T.D. Dzhuraev [3] and U.I. Baltaeva [4], [5] are insufficient for equation (1.1) as $f_{i}(x, y ; u(x, 0)) \neq 0$ and $0<\alpha<1$. This is mostly related with the fractional differential operator, which is the Caputo operator in our case, and the arising integral equations and the methods for studying them are related with a nonlinear part of the considered equation.

Local and nonlocal problems with continuous and integral conjugate conditions for loaded parabolic hyperbolic second order equations involving various operator like Caputo operator, Riemann-Liouville operator and others, were studied in works [6]-8].

We note that the aforementioned equations describe some problems of optimal control, governing of ground water, the moisture of soil, problems on underground liquids, problems in gas dynamics, mathematical biology, economics, ecology and fundamental mathematics [9]-[12]. Moreover, boundary value problems for differential and integral-differential equations with nonlocal boundary conditions arise in various fields of mechanics, physics, biology, biotechnology and others, see [13]-[15].

First we provide some conditions necessary for formulation of problems related with possible cases of choice of the coefficients $a, b$ :

$$
\begin{array}{rlrl}
u(0, y) & =\varphi_{1}(y), & & u(1, y)=\varphi_{2}(y), \quad 0 \leqslant y \leqslant h, \\
u_{x x}(0, y) & =\varphi_{3}(y), \quad 0 \leqslant y \leqslant h, & \\
u_{x x}(1, y) & =\varphi_{4}(y), \quad 0 \leqslant y \leqslant h, \\
\frac{d}{d x} u(\theta(x)) & =a_{1}(x) u_{y}(x,-0)+a_{2}(x) u_{x}(x,-0)+a_{3}(x) u(x, 0)+a_{4}(x), \quad 0 \leqslant x<1, \\
\left.\frac{\partial u}{\partial n}\right|_{A C} & =\psi_{1}(x), & & 0 \leqslant x \leqslant \frac{1}{2}, \\
\left.\frac{\partial u}{\partial n}\right|_{B C} & =\psi_{2}(x), & & \frac{1}{2} \leqslant x \leqslant 1,
\end{array}
$$

where $n$ is an inward normal, $\theta(x)=\theta\left(\frac{x}{2},-\frac{x}{2}\right), \varphi_{j}(y), a_{j}(x), j=\overline{1,4}, \psi_{i}(x), i=\overline{1,2}$, are given functions. 


\section{Formulation OF PROBLEM}

Definition 2.1. A function $u(x, y)$ is called a regular solution of equation (1.1) if it possesses continuous derivatives involved in the operator $L u$ and $L u \in C^{1}(\Omega \backslash A B)$.

Problem I. Find a regular solution $u(x, y)$ of equation 1.1 in the domain $\Omega \backslash A B$ possessing the following properties:

1) $u(x, y) \in C(\bar{\Omega}) \cap C^{1}\left(\overline{\Omega_{2}} \backslash \overline{B C}\right), \quad u_{x x} \in C\left(\Omega_{1} \cup A A_{0}\right)$;

2) $u(x, y)$ satisfies boundary conditions $(1.3),(1.4),(1.6)$ and 1.7 ;

3) an integral conjugate condition

$$
\begin{aligned}
\lim _{y \rightarrow+0}{ }_{c} D_{0 y}^{\alpha} u(x, y)= & \lambda_{1}(x) u_{y}(x,-0)+\lambda_{2}(x) u_{x}(x,-0)+\lambda_{3}(x) u(x,-0) \\
& +\lambda_{4}(x) \int_{0}^{x} r(t) u(t, 0) d t+\lambda_{5}(x)
\end{aligned}
$$

is satisfied, where $\lambda_{i}(x), i=\overline{1,5}$, are given functions and

$$
\sum_{i=1}^{4} \lambda_{i}^{2}(x) \neq 0
$$

\section{STUdy OF PROBLEM I.}

We denote:

$$
u(x, y)= \begin{cases}u_{1}(x, y), & (x, y) \in \Omega_{1}, \\ u_{2}(x, y), & (x, y) \in \Omega_{2} .\end{cases}
$$

Then equation 1.1 can be rewritten as two systems:

$$
\left\{\begin{array}{rlrl}
u_{1 x x}-{ }_{c} D_{0 y}^{\alpha} u_{1}+f_{1}\left(x, y ; u_{1}(x, 0)\right) & =v_{1}(x, y), & \\
a v_{1 x}+b v_{1 y}+c v_{1} & =0, & & (x, y) \in \Omega_{1}
\end{array}\right.
$$

and

$$
\left\{\begin{array}{rl}
u_{2 x x}-u_{2 y y}+f_{2}\left(x, y ; u_{2}(x, 0)\right) & =v_{2}(x, y), \\
a v_{2 x}+b v_{2 y}+c v_{2} & =0,
\end{array} \quad(x, y) \in \Omega_{2},\right.
$$

where $v_{i}(x, y) \in C^{1}\left(\Omega_{i}\right), i=1,2$, are sufficiently smooth functions.

Let $0<b / a \leqslant 1$, then, assuming that $a>0, b>0$, we obtain:

$$
0<b \leqslant \frac{b+a}{2}, \quad 0 \leqslant b x-a y \leqslant \frac{b+a}{2}, \quad(x, y) \in \Omega_{2} .
$$

We note that a general solution to the equation

$$
a v_{i x}+b v_{i y}+c v_{i}=0, \quad(i=1,2)
$$

reads as

$$
v_{i}(x, y)=w_{i}(b x-a y) \exp \left[-\frac{c}{2 a b}(b x+a y)\right],
$$

where $w_{i}(b x-a y), i=1,2$, are arbitrary continuously differentiable functions.

On the base of (3.3), (3.1) and (3.2), we consider the following equations:

$$
\begin{aligned}
& u_{1 x x}-{ }_{c} D_{0 y}^{\alpha} u_{1}+f_{1}\left(x, y ; u_{1}(x, 0)\right)=w_{1}(b x-a y) \exp \left(-\frac{c}{2 a b}(b x+a y)\right), \\
& u_{2 x x}-u_{2 y y}+f_{2}\left(x, y ; u_{2}(x, 0)\right)=w_{2}(b x-a y) \exp \left(-\frac{c}{2 a b}(b x+a y)\right) .
\end{aligned}
$$


It is easy to observe that the solution to the Cauchy problem for equation (3.5) with initial conditions $u(x,-0)=\tau(x), \quad u_{y}(x,-0)=\nu^{-}(x)$ in the domain $\Omega_{2}$ reads as

$$
\begin{aligned}
u_{2}(x, y)= & \frac{\tau(x+y)+\tau(x-y)}{2}+\frac{1}{2} \int_{x-y}^{x+y} \nu^{-}(t) d t \\
& -\frac{1}{4} \int_{x+y}^{x-y} d \xi \int_{\xi}^{x-y} f_{2}\left(\frac{\xi+\eta}{2}, \frac{\xi-\eta}{2} ; \tau\left(\frac{\xi+\eta}{2}\right)\right) d \eta \\
& +\frac{1}{4} \int_{x+y}^{x-y} d \xi \int_{\xi}^{x-y} w_{2}\left(\frac{b-a}{2} \xi+\frac{b+a}{2} \eta\right) \exp \left(\frac{-c}{2 a b}\left(\frac{b+a}{2} \xi+\frac{b-a}{2} \eta\right)\right) d \eta .
\end{aligned}
$$

Taking into consideration that $\left.\left(u_{x}+u_{y}\right)\right|_{A C}=\sqrt{2} \psi_{1}(x)$ and employing conditions (1.6) and (1.7), by (3.6) we find:

$$
\begin{aligned}
\left(2 a_{1}(x)+1\right) \nu^{-}(x)= & \left(1-2 a_{2}(x)\right) \tau^{\prime}(x)-\frac{1}{2} \int_{0}^{x} f_{2}\left(\frac{\xi+x}{2}, \frac{\xi-x}{2} ; \tau\left(\frac{\xi+x}{2}\right)\right) d \xi-2 a_{3}(x) \tau(x) \\
& +\frac{1}{2} \int_{0}^{x} w_{2}\left(\frac{b-a}{2} \xi+\frac{b+a}{2} x\right) e^{\frac{-c}{2 a b}\left(\frac{b+a}{2} \xi+\frac{b-a}{2} x\right)} d \xi-2 a_{4}(x)
\end{aligned}
$$

and

$$
w_{2}(x)=\left(f_{2}\left(\frac{x}{a+b},-\frac{x}{a+b} ; \tau\left(\frac{x}{a+b}\right)\right)-\sqrt{2} \psi_{1}^{\prime}\left(\frac{x}{a+b}\right)\right) e^{\frac{c(b-a) x}{2 a b(a+b)}} .
$$

On the other hand, by $(1.4)$ and

$$
{ }_{c} D_{0 y}^{\alpha} u_{1}(0, y)={ }_{c} D_{0 y}^{\alpha} \varphi_{1}(y), \quad \tau(0)=\varphi_{1}(0)
$$

as $x \rightarrow+0$, it follows from $(3.4)$ that

$$
w_{1}(-a y)=\left[\varphi_{3}(y)-{ }_{c} D_{o y}^{\alpha} \varphi_{1}(y)+f_{1}\left(0, y ; \varphi_{1}(0)\right)\right] \exp \left(\frac{c y}{2 b}\right),
$$

that is,

$$
w_{1}(y)=\left(\varphi_{3}\left(\frac{-y}{a}\right)-{ }_{c} D_{o y}^{\alpha} \varphi_{1}\left(\frac{-y}{a}\right)+f_{1}\left(0,-\frac{y}{a} ; \varphi_{1}(0)\right)\right) \exp \left(-\frac{c y}{2 a b}\right) .
$$

\subsection{Main result.}

Theorem 3.1. If conditions

$$
\begin{aligned}
& \varphi_{1}(y) \in C^{2}(0, h) \cap C^{1}[0, h], \quad \varphi_{2}(y), \varphi_{3}(y) \in C^{1}(0, h) \cap C[0, h] ; \\
& a_{j}(x) \in C[0,1] \cap C^{2}(0,1), \quad \lambda_{j}(x), \lambda_{5}(x) \in C[0,1] \cap C^{1}(0,1), \quad j=1,4 ; \\
& \psi_{1}(x) \in C^{2}\left(0, \frac{1}{2}\right) \cap C^{1}\left[0, \frac{1}{2}\right], \quad f_{i}(x, y ; u(x, 0)) \in C\left(\bar{\Omega}_{i}\right) \cap C^{1}\left(\Omega_{i}\right), \quad i=1,2 ; \\
&\left|f_{i}\left(x, y ; \tau_{1}(x)\right)-f_{i}\left(x, y ; \tau_{2}(x)\right)\right| \leqslant L_{i}\left|\tau_{1}(x)-\tau_{2}(x)\right|, \quad i=1,2,
\end{aligned}
$$

are satisfied, where $L_{i}=$ const $>0, i=1,2$, then a solution to Problem I exists and is unique. 
Proof. In view of our notations,

$$
u(x,-0)=u(x,+0)=\tau(x), \quad u_{y}(x, 0)=\nu^{-}(x), \quad \lim _{y \rightarrow+0}{ }_{c} D_{0 y}^{\alpha} u=\nu^{+}(x)
$$

as $y \rightarrow+0$, by $(3.4)$ and conjugate condition $(2.1)$ we have that

$$
\begin{aligned}
\tau^{\prime \prime}(x)-\lambda_{1}(x) \nu^{-}(x)-\lambda_{2}(x) \tau^{\prime}(x) & -\lambda_{3}(x) \tau(x)-\lambda_{4}(x) \int_{0}^{x} r(t) \tau(t) d t \\
& +f_{1}(x, 0 ; \tau(x))=w_{1}(b x) e^{-\frac{c x}{2 a}}+\lambda_{5}(x) .
\end{aligned}
$$

Employing 3.7 and (3.15), after simplifications, as $2 a_{1}(x)+1 \neq 0$ we get:

$$
\begin{array}{r}
\tau^{\prime \prime}(x)+A_{1}(x) \tau^{\prime}(x)+B_{1}(x) \tau(x)+C_{1}(x) \int_{0}^{x} f_{2}\left(\frac{\xi+x}{2}, \frac{\xi-x}{2} ; \tau\left(\frac{\xi+x}{2}\right)\right) d \xi \\
+f_{1}(x, 0 ; \tau(x))+\lambda_{4}(x) \int_{0}^{x} r(t) \tau(t) d t=f(x),
\end{array}
$$

where

$$
\begin{aligned}
A_{1}(x) & =-2 C_{1}(x)\left(2 a_{2}(x)-1\right)+\lambda_{2}(x), \\
B_{1}(x) & =-4 C_{1}(x) a_{3}(x)+\lambda_{3}(x), \\
C_{1}(x)= & \frac{-\lambda_{1}(x)}{2\left(1+2 a_{1}(x)\right)}, \\
f(x)= & -\frac{\lambda_{1}(x)}{2\left(1+2 a_{1}(x)\right)} \int_{0}^{x} w_{2}\left(\frac{b-a}{2} \xi+\frac{b+a}{2} x\right) e^{\frac{-c}{2 a b}\left(\frac{b+a}{2} \xi+\frac{b-a}{2} x\right)} d \xi \\
& -\frac{\lambda_{1}(x) a_{4}(x)}{1+2 a_{1}(x)}+w_{1}(b x) e^{\frac{-c x}{2 a}}+\lambda_{5}(x) .
\end{aligned}
$$

Assuming $a \neq b$ and making the change $\frac{b-a}{2} \xi+\frac{b+a}{2} x=t$, we can rewrite the function $f(x)$ in the form:

$$
\begin{aligned}
f(x)= & \frac{\lambda_{1}(x)}{(a-b)\left(1+2 a_{1}(x)\right)} \int_{\frac{b+a}{2} x}^{b x} w_{2}(t) e^{\frac{c(a+b)}{2 a b(a-b)} t+\frac{c}{b-a} x} d t \\
& -\frac{\lambda_{1}(x) a_{4}(x)}{1+2 a_{1}(x)}+w_{1}(b x) e^{\frac{-c x}{2 a}}+\lambda_{5}(x) .
\end{aligned}
$$

Then in view of (3.8), after some simplifications we finally find:

$$
\begin{aligned}
f(x)= & \frac{(a+b) \lambda_{1}(x)}{(a-b)\left(1+2 a_{1}(x)\right)} \int_{\frac{x}{2}}^{\frac{b x}{a+b}} e^{\frac{c(2 t-x)}{(a-b)}}\left(f_{2}(t,-t ; \tau(t))-\sqrt{2} \psi_{1}^{\prime}(t)\right) d t \\
& -\frac{\lambda_{1}(x) a_{4}(x)}{1+2 a_{1}(x)}+w_{1}(b x) e^{\frac{-c x}{2 a}}+\lambda_{5}(x) .
\end{aligned}
$$

Taking into consideration that

$$
\tau(0)=\varphi_{1}(0), \quad \tau^{\prime}(0)=\sqrt{2} \psi_{1}(0)-\varphi_{1}^{\prime}(0)
$$


and integrating twice 3.16$)$ with respect to $x$ from 0 to $x$, we obtain:

$$
\begin{aligned}
\tau(x) & +\int_{0}^{x}\left[(x-t)\left(B_{1}(t)-A_{1}^{\prime}(t)\right)+A_{1}(t)\right] \tau(t) d t+\int_{0}^{x} \tau(t) d t \int_{t}^{x} r(t)(x-z) \lambda_{4}(z) d z \\
& +\int_{0}^{x}(x-t) f_{1}(t, 0 ; \tau(t)) d t+2 \int_{0}^{x}(x-t) C_{1}(t) d t \int_{t / 2}^{t} f_{2}(s, s-t ; \tau(s)) d s \\
& -\frac{2(a+b)}{a-b} \int_{0}^{x}(x-t) C_{1}(t) d t \int_{t / 2}^{\frac{b t}{a+b}} e^{\frac{c(2 s-t)}{a-b}} f_{2}(s,-s ; \tau(s)) d s=\int_{0}^{x}(x-t)\left(w_{1}(b t) e^{-\frac{c t}{2 a}}+\lambda_{5}(t)\right) d t \\
& -\frac{2 \sqrt{2}(a+b)}{a-b} \int_{0}^{x}(x-t) C_{1}(t) d t \int_{t / 2}^{\frac{b t}{a+b}} e^{\frac{c(2 s-t)}{a-b}} \psi_{1}^{\prime}(s) d s+x\left(\varphi_{1}(0) A_{1}(0)+\sqrt{2} \psi_{1}(0)-\varphi_{1}^{\prime}(0)\right) \\
+ & \psi_{1}(0)-\int_{0}^{x}(x-t) \frac{\lambda_{1}(t) a_{4}(t)}{1+2 a_{2}(t)} d t .
\end{aligned}
$$

Thus, we have obtained a Volterra type nonlinear integral equation:

$$
\begin{aligned}
\tau(x)= & 2 \int_{0}^{x}(x-t)\left(\frac{(a+b)}{a-b} \int_{\frac{t}{2}}^{\frac{b t}{a+b}} e^{\frac{c(2 s-t)}{a-b}} f_{2}(s,-s ; \tau(s)) d s-\int_{\frac{t}{2}}^{t} f_{2}(s, s-t ; \tau(s)) d s\right) C_{1}(t) d t \\
& -\int_{0}^{x} K_{1}(x, t) \tau(t) d t-\int_{0}^{x}(x-t) f_{1}(t, 0 ; \tau(t)) d t+f^{*}(x),
\end{aligned}
$$

where

$$
\begin{aligned}
K_{1}(x, t)= & (x-t)\left(B_{1}(t)-A_{1}^{\prime}(t)\right)+A_{1}(t)+r(t) \int_{t}^{x}(x-z) \lambda_{4}(z) d z, \\
f^{*}(x)= & \int_{0}^{x}(x-t)\left(w_{1}(b t) e^{-\frac{c t}{2 a}}-\frac{\lambda_{1}(t) a_{4}(t)}{1+2 a_{2}(t)}+\lambda_{5}(t)\right) d t+x\left(\varphi_{1}(0) A_{1}(0)+\sqrt{2} \psi_{1}(0)-\varphi_{1}^{\prime}(0)\right) \\
& -\frac{2 \sqrt{2}(a+b)}{a-b} \int_{0}^{x}(x-t) C_{1}(t) d t \int_{t / 2}^{\frac{b t}{a+b}} e^{\frac{c(2 s-t)}{a-b}} \psi_{1}^{\prime}(s) d s+\psi_{1}(0) .
\end{aligned}
$$

By $3.10-3.12$ we hence get:

$$
\left\|K_{1}(x, t)\right\|_{C} \leqslant M, \quad 0 \leqslant t \leqslant x \leqslant 1 ; \quad\left\|f^{*}(x)\right\|_{C} \leqslant f_{0}, \quad 0 \leqslant x \leqslant 1,
$$

wnere $M, f_{0}=$ const $>0$. In view of inequalities

$$
\left\|f_{i}(x, y ; \tau(x))\right\|_{C} \leqslant f_{0 i}, \quad\left\|C_{1}(x)\right\|_{C} \leqslant c_{0}
$$


and assuming $\tau_{0}(x)=f^{*}(x)$, by

$$
\begin{aligned}
\tau_{n}(x)= & \frac{2(a+b)}{a-b} \int_{0}^{x}(x-t) C_{1}(t) d t \int_{\frac{t}{2}}^{\frac{b t}{a+b}} e^{\frac{c(2 s-t)}{a-b}} f_{2}\left(s,-s ; \tau_{n-1}(s)\right) d s \\
& -2 \int_{0}^{x}(x-t) C_{1}(t) d t \int_{\frac{t}{2}}^{t} f_{2}\left(s, s-t ; \tau_{n-1}(s)\right) d s \\
& -\int_{0}^{x} K_{1}(x, t) \tau_{n-1}(t) d t-\int_{0}^{x}(x-t) f_{1}\left(t, 0 ; \tau_{n-1}(t)\right) d t+f^{*}(x),
\end{aligned}
$$

we obtain:

$$
\left\|\tau_{1}(x)-\tau_{0}(x)\right\|_{C} \leqslant x \cdot c_{01},
$$

where $f_{0}, c_{0}, c_{01}=$ const $>0$, and

$$
c_{01}=\frac{1}{4} \cdot \max \left\{\frac{(a+b) c_{0} f_{02}}{a-b} ; \frac{c_{0} f_{02}}{3} ; M f_{0} ; \frac{f_{01}}{2}\right\} .
$$

Then by 3.13), 3.22, 3.21 we find:

$$
\begin{aligned}
& \left\|\tau_{2}(x)-\tau_{1}(x)\right\|_{C} \leqslant x^{2} \cdot c_{11}+x^{3} \cdot c_{12}+x^{4} \cdot c_{13} \leqslant \frac{x^{2}}{2 !} \cdot c_{01} m \\
& \left\|\tau_{3}(x)-\tau_{2}(x)\right\|_{C} \leqslant \frac{x^{3}}{3 !} \cdot c_{21}+\frac{x^{4}}{4 !} \cdot c_{22}+\frac{x^{5}}{5 !} \cdot c_{23} \leqslant \frac{x^{3}}{3 !} \cdot c_{01} m^{2}
\end{aligned}
$$

where

$$
\begin{aligned}
c_{11} & =c_{01} \cdot \frac{M}{2}, \quad c_{12}=c_{01} \cdot \frac{L_{1}}{6}, \quad c_{13}=c_{01} \cdot \frac{c_{0} L_{2}(2 a+b)}{a-b}, \\
c_{21} & =m \cdot c_{11}, \quad c_{22}=m \cdot c_{12}, \quad c_{23}=m \cdot c_{13}, \\
m & =\frac{1}{3} \cdot \max \left\{\frac{M}{2} ; \frac{L_{1}}{6} ; \frac{c_{0} L_{2}(2 a+2)}{a-b}\right\} .
\end{aligned}
$$

Thus, we finally have:

$$
\left\|\tau_{n}(x)-\tau_{n-1}(x)\right\|_{C} \leqslant c_{01} m^{n-1} \cdot \frac{x^{n}}{n !} .
$$

Due to 3.23 we conclude that equation 3.19 involves a contracting operator and there exists a unique fixed point of this operator. Therefore, a nonlinear integral equation (3.19) possesses a unique solution in the class $C[0,1] \cap C^{2}(0,1)$.

Remark 3.1. If $a=b$, then we take into consideration that by (3.17) we have

$$
f(x)=\frac{a \lambda_{1}(x)}{c\left(1+2 a_{1}(x)\right)} w_{2}(a x)\left(e^{\frac{-c x}{2 a}}-1\right)-\frac{\lambda_{1}(x) a_{4}(x)}{1+2 a_{1}(x)}+w_{1}(a x) e^{\frac{-c x}{2 a}}+\lambda_{5}(x)
$$

and

$$
w_{2}(x)=f_{2}\left(\frac{x}{2 a},-\frac{x}{2 a} ; \tau\left(\frac{x}{2 a}\right)\right)-\sqrt{2} \psi_{1}^{\prime}\left(\frac{x}{2 a}\right),
$$


by (3.16) we similarly obtain a nonlinear integral Volterra equation:

$$
\begin{aligned}
\tau(x)= & \frac{4 a}{c} \int_{0}^{\frac{x}{2}}(x-2 t) C_{1}(2 t)\left(e^{\frac{-c t}{a}}-1\right) f_{2}(t,-t ; \tau(t)) d t-2 \int_{0}^{x}(x-t) C_{1}(t) d t \int_{t / 2}^{t} f_{2}(s, s-t ; \tau(s)) d s \\
& -\int_{0}^{x} K_{1}(x, t) \tau(t) d t-\int_{0}^{x}(x-t) f_{1}(t, 0 ; \tau(t)) d t+f_{1}^{*}(x),
\end{aligned}
$$

where

$$
\begin{aligned}
f_{1}^{*}(x)= & x\left(\varphi_{1}(0) A_{1}(0)+\sqrt{2} \psi_{3}(0)-\varphi_{1}^{\prime}(0)\right)+\int_{0}^{x}(x-t)\left(w_{1}(b t) e^{-\frac{c t}{2 a}}+\lambda_{5}(t)-\frac{\lambda_{1}(t) a_{4}(t)}{1+2 a_{2}(t)}\right) d t \\
& -\frac{4 \sqrt{2} a}{c} \int_{0}^{\frac{x}{2}}(x-2 t) C_{1}(2 t)\left(e^{\frac{-c t}{a}}-1\right) \psi_{1}^{\prime}(t) d t+\psi_{1}(0) .
\end{aligned}
$$

Then $\nu^{-}(x)$ can be found by (3.7) and hence, a solution of the considered problem in the domain $\Omega_{2}$ is recovered as a solution of a Cauchy problem, see (3.6).

Remark 3.2. In the cases $2 a_{1}(x)+1=0,2 a_{2}(x)-1 \neq 0$ and $2 a_{1}(x)+1=0, a_{3}(x) \neq 0$ the unknown function $\tau(x)$ is determined as a solution to a nonlinear integral Volterra equation, which is implied by (3.7).

The solutions of the studied problem in the domain $\Omega_{2}$ is recovered in the same way, see (3.6), while in the domain $\Omega_{1}$ it is found a solution to the Dirichlet problem for equation (3.4) [16]:

$$
\begin{aligned}
u_{1}(x, y)= & \int_{0}^{y} G_{\xi}(x, y, 0, \eta) \varphi_{1}(\eta) d \eta-\int_{0}^{y} G_{\xi}(x, y, 1, \eta) \varphi_{2}(\eta) d \eta+\int_{0}^{1} G_{0}(x, \xi, y) \tau(\xi) d \xi \\
& -\int_{0}^{y} \int_{0}^{1} G(x, y, \xi, \eta)\left(\omega_{1}(b \xi-a \eta) e^{-\frac{c(b \xi+a \eta)}{2 a b}}-f_{1}(\xi, \eta ; \tau(\xi))\right) d \xi d \eta,
\end{aligned}
$$

where $\omega_{1}(\cdot)$ is determined by $(3.9)$ and

$$
\begin{aligned}
& G_{0}(x, \xi, y)=\frac{1}{\Gamma(1-\alpha)} \int_{0}^{y} \eta^{-\alpha} G(x, y, \xi, \eta) d \eta \\
& G(x, y, \xi, \eta)=\frac{(y-\eta)^{\frac{\alpha}{2}}}{2} \sum_{n=-\infty}^{\infty}\left[e_{1, \frac{\alpha}{2}}^{1, \frac{\alpha}{2}}\left(-\frac{|x-\xi+2 n|}{(y-\eta)^{\frac{\alpha}{2}}}\right)-e_{1, \frac{\alpha}{2}}^{1, \frac{\alpha}{2}}\left(-\frac{|x+\xi+2 n|}{(y-\eta)^{\frac{\alpha}{2}}}\right)\right]
\end{aligned}
$$

is the Green function of the Dirichlet problem,

$$
e_{1, \delta}^{1, \delta}=\sum_{n=0}^{\infty} \frac{z^{n}}{n ! \Gamma(\delta-\delta n)}
$$

is a Wright type function [16].

It should be noted that in the cases $2 a_{1}(x)+1=0,2 a_{2}(x)-1 \neq 0$ and $2 a_{1}(x)+1=0$, $a_{3}(x) \neq 0$ we first find $\nu^{+}(x)$ via a solution to the Dirichlet problem, see (3.24), and then we employ the conjugate conditions to find $\nu^{-}(x)$ as $\lambda_{1}(x) \neq 0$. 


\section{OTHER PROBLEMS RELATED WITH CONDITIONS ON $a$ AND $b$}

Problme II. Find a regular solution $u(x, y)$ of equation $(1.1)$ in the domain $\Omega \backslash A B$ with the following properties:

1) $u(x, y) \in C(\bar{\Omega}) \cap C^{1}\left(\overline{\Omega_{2}}\right), \quad u_{x x} \in C\left(\Omega_{1} \cup A A_{0}\right)$;

2) $u(x, y)$ satisfies all conditions of Problem I and (1.8) for $1<b / a<+\infty$.

Remark 4.1. For a well-posed formulation of the problem we find appropriate conditions for determining an unknown function $w_{i}(b x-a y), i=1,2$, the domain of which covers completely the considered domain under approrpriate conditions for the coefficients $a$ and $b$. In order to be able to do this, Problem II involves additional condition (1.8).

Problem III. Find a regular solution $u(x, y) \in C(\bar{\Omega}) \cap C^{1}\left(\overline{\Omega_{2}}\right), u_{x x} \in C\left(\Omega_{1} \cup B B_{0}\right)$ for 1.1$)$ in the domain $\Omega \backslash A B$ satisfying all conditions of Problem II, when (1.4) is replaced by (1.5) for $-\infty<b / a<-1$.

Problem IV. Find a regular solution

$$
u(x, y) \in C(\bar{\Omega}) \cap C^{1}\left(\overline{\Omega_{2}} \backslash \overline{A C}\right), \quad u_{x x} \in C\left(\Omega_{1} \cup B B_{0}\right)
$$

to equation $(1.1)$ in the domain $\Omega \backslash A B$ satisfying all conditions of Problem III except for (1.7) as $-1 \leqslant b / a<0$.

Remark 4.2. In view of the conditions for the coefficients a and b, for a well-posed formulation of Problems III and IV, in contrast to Problems I and II, a condition for determining unknown functions $w_{i}(b x-a y), i=1,2$, is posed on the segment $x=1$.

4.1. Study of Problem II. Let $1<b / a<+\infty$, then, assuming $a>0$ and $b>0$, we have $b>(a+b) / 2$ and therefore,

$$
\begin{array}{ll}
0 \leqslant b x-a y \leqslant \frac{b+a}{2}, & (x, y) \in \Omega_{21}, \\
\frac{b+a}{2} \leqslant b x-a y \leqslant b, & (x, y) \in \Omega_{22} .
\end{array}
$$

Here $\Omega_{21}$ and $\Omega_{22}$ are the characteristic triangles $A B E$ and $B C E$, respectively, where $E=$ $E\left(\frac{b+a}{2 b}, 0\right)$, and $\Omega_{21} \cup C E \cup \Omega_{22}=\Omega_{2}$.

Assume that inequality (4.1) holds, then, applying (1.6) and (1.7), from the solution (3.6) we find functional relation (3.7) and $\omega_{21}(x)$, see (3.8):

$$
w_{21}((a+b) x)=e^{\frac{c(b-a) x}{2 a b}}\left(f_{2}(x,-x ; \tau(x))-\sqrt{2} \psi_{1}^{\prime}(x)\right) .
$$

In case 4.2 we employ condition $(1.8)$ and $\left.\left(u_{y}-u_{x}\right)\right|_{B C}=\sqrt{2} \psi_{2}(x)$ from $(3.6)$ to find $w_{22}(x)$ :

$$
w_{22}((b-a) x+a)=e^{\frac{c}{2 a b}((b+a) x-a)}\left(\sqrt{2} \psi_{2}^{\prime}(x)+f_{2}(x, x-1 ; \tau(x))\right) .
$$

It should be noted that the function

$$
w_{2}(x)= \begin{cases}w_{21}(x), & 0 \leqslant x \leqslant \frac{a+b}{2} \\ w_{22}(x), & \frac{a+b}{2} \leqslant x \leqslant b\end{cases}
$$


should be continuous for all $0<x<b$. It is easy to confirm that

$$
w_{21}\left(\frac{a+b}{2}\right)=w_{22}\left(\frac{a+b}{2}\right)
$$

as

$$
\psi_{1}^{\prime}\left(\frac{1}{2}\right)=-\psi_{2}^{\prime}\left(\frac{1}{2}\right)
$$

By $4.5,3.7$ and 3.15 we find:

$$
\begin{aligned}
\tau(x)= & \Phi(x, \tau(x))-2 \int_{0}^{x}(x-t) C_{1}(t) d t \int_{t / 2}^{t} f_{2}(s, s-t ; \tau(s)) d s \\
& -\int_{0}^{x} K_{1}(x, t) \tau(t) d t-\int_{0}^{x}(x-t) f_{1}(t, 0 ; \tau(t)) d t+g^{*}(x),
\end{aligned}
$$

where

$$
\begin{aligned}
& \Phi(x, \tau(x))=\frac{2}{a-b} \int_{0}^{\frac{a+b}{2}} w_{21}(z) K_{2}(x, z) d z \\
& +\frac{2}{a-b} \int_{\frac{a+b}{2}}^{b x} w_{22}(z) e^{-\frac{c(a+b) z}{2 a b(b-a)}} d z \int_{z / 2}^{x}(x-t) C_{1}(t) e^{\frac{c t}{2(b-a)}} d t, \\
& K_{2}(x, z)=\left\{\begin{array}{cl}
e^{-\frac{c(a+b) z}{2 a b(b-a)}} \int_{\frac{z}{2}}^{\frac{2 z}{b+a}}(x-t) C_{1}(t) e^{\frac{c t}{2(b-a)}} d t, & 0 \leqslant z \leqslant \frac{a+b}{2} x ; \\
e^{-\frac{c(a+b) z}{2 a b(b-a)}} \int_{z / 2}^{x}(x-t) C_{1}(t) e^{\frac{c t}{2(b-a)}} d t, & \frac{a+b}{2} x \leqslant z \leqslant \frac{a+b}{2},
\end{array}\right. \\
& g^{*}(x)=\int_{0}^{x}(x-t)\left(w_{1}(b t) e^{-\frac{c t}{2 a}}+\lambda_{5}(t)-\frac{\lambda_{1}(t) a_{4}(t)}{1+2 a_{2}(t)}\right) d t \\
& +x\left(\varphi_{1}(0) A_{1}(0)+\sqrt{2} \psi_{3}(0)-\varphi_{1}^{\prime}(0)\right)+\psi_{1}(0) .
\end{aligned}
$$

Substituting (4.3) and (4.4) into 4.7), we obtain:

$$
\begin{aligned}
\Phi(x, \tau(x))= & \frac{2}{a-b} \int_{0}^{\frac{a+b}{2}} e^{\frac{c(b-a) z}{2 a b(a+b)}} f_{2}\left(\frac{z}{a+b},-\frac{z}{a+b} ; \tau\left(\frac{z}{a+b}\right)\right) K_{2}(x, z) d z \\
& +\frac{2}{a-b} \int_{\frac{a+b}{2}}^{b x} e^{\frac{-c}{b-a}} f_{2}\left(\frac{z-a}{b-a}, \frac{z-a}{b-a}-1 ; \tau\left(\frac{z-a}{b-a}\right)\right) d z \int_{z / 2}^{x}(x-t) C_{1}(t) e^{\frac{c t}{2(b-a)}} d t \\
= & \frac{2(a+b)}{a-b} \int_{0}^{\frac{1}{2}} e^{\frac{c(b-a) z}{2 a b}} f_{2}(z,-z ; \tau(z)) K_{2}(x, z(a+b)) d z
\end{aligned}
$$




$$
-2 e^{\frac{-c}{b-a}} \int_{\frac{1}{2}}^{\frac{b x-a}{b-a}} f_{2}(z, z-1 ; \tau(z)) d z \int_{\frac{z(b-a)+a}{2}}^{x}(x-t) C_{1}(t) e^{\frac{c t}{2(b-a)}} d t .
$$

Moreover, taking into consideration that

$$
\left\|\int_{\frac{z(b-a)+a}{2}}^{x}(x-t) C_{1}(t) e^{\frac{c t}{2(b-a)}} d t\right\|_{C} \leqslant \text { const and }\left\|K_{2}(x, z)\right\|_{C} \leqslant \text { const, }
$$

we conclude that

$$
\|\Phi(x, \tau(x))\|_{C} \leqslant m_{1}\left\|\int_{0}^{\frac{1}{2}} f_{2}(z,-z ; \tau(z)) d z\right\|_{C}+m_{2}\left\|\int_{\frac{1}{2}}^{\frac{b x-a}{b-a}} f_{2}(z, z-1 ; \tau(z)) d z\right\|_{C},
$$

where $m_{1}, m_{2}=$ const $>0$.

By (3.13) and the second condition in 3.12 , assuming that $m_{0}=\max \left\{m_{1}, m_{2}\right\}$, we get:

$$
\begin{aligned}
&\|\Phi(x, \tau(x))\|_{C} \leqslant \frac{b x-a}{b-a} \cdot \text { const } \\
&\left\|\Phi\left(x, \tau_{n-1}(x)\right)-\Phi\left(x, \tau_{n-2}(x)\right)\right\|_{C} \leqslant m L_{2}\left\|\int_{0}^{\frac{b x-a}{b-a}}\left|\tau_{n-1}(z)-\tau_{n-2}(z)\right| d z\right\|_{C} \\
& \leqslant \frac{m_{0} L_{2}}{n !}\left(\frac{b x-a}{b-a}\right)^{n} \cdot \text { const. }
\end{aligned}
$$

Using the inequality $x<\frac{b x-a}{b-a}$ as $b>a$, by similar arguing for equation 4.6 we get:

$$
\left\|\tau_{n}(x)-\tau_{n-1}(x)\right\|_{C} \leqslant \frac{(b x-a)^{n}}{(b-a)^{n} n !} \cdot \text { const. }
$$

Thus, we conclude that equation (4.6) is uniquely solvable as a Volterra nonlinear integral equation.

The following theorem holds.

Theorem 4.1. If the assumptions of Theorem 3.1 and

$$
\psi_{2}(x) \in C^{2}\left(\frac{1}{2}, 1\right) \cap C^{1}\left[\frac{1}{2}, 1\right],
$$

then Problem II is uniquely solvable.

Remark 4.3. While studying Problem II, the unknown function $\tau(x)$ can be determined as a solution to the nonlinear integral equation as $2 a_{1}(x)+1=0,2 a_{2}(x)-1 \neq 0$, or $2 a_{1}(x)+1=0$, $a_{3}(x) \neq 0$, in the same way as in Problem I.

Remark 4.4. Problems III and $I V$ are reduced to the Cauchy problem for equation (3.16) with initial conditions $\tau(1)=\varphi_{2}(0), \tau^{\prime}(1)=\varphi_{2}^{\prime}(0)-\sqrt{2} \psi_{2}(0)$.

Let $-1 \leqslant \frac{b}{a}<0$, then the unknown function $w_{2}(x)$ is determined by condition 1.8 and is given by 4.4 ). In the other case, that is, as $-\infty<\frac{b}{a}<-1$, the function $w_{2}(x)$ is determined by 4.5). It should be noted that in these case we obtain a linear integral Fredholm type equation as $f_{i}(x, y ; \tau(x))=0$ and $\lambda_{4}(x) \neq 0$. Therefore, we need to prove independently the uniqueness 
of solution to the problem or to impose additional conditions for the given functions ensuring the unique solvability of the corresponding linear integral Fredhold type equation. But since $f_{i}(x, y ; \tau(x)) \neq 0, i=1,2$, we obtain a nonlinear integral equation with Fredholm and Volterra operators.

On the other hand, if we replace the integral term in conjugate condition (2.1) by

$$
\lambda_{4}(x) \int_{x}^{1} r(t) \tau(t) d t
$$

and the corresponding nonlocal condition, see (1.6), is imposed on the characteristics $B C$, then we again obtain a nonlinear Volterra type integral equation.

Remark 4.5. Similar problems for 1.1 as $b=0, a, c \neq 0$ can be studied by a similar method.

Such problems were studied in work [17] as

$$
f_{i}(x, y ; u(x, 0))=\sum_{k=1}^{n} p_{k} I_{0 x}^{\beta_{i k}} u(x ; 0), \quad i=1,2 .
$$

As we know, boundary value problems for equation (1.1) with $a=0, b, c \neq 0$ were not studied even as $f_{i}(x, y ; u(x, 0))=0$. We note that the methods used in work [3] for $a=0, b, c \neq 0$, are not applicable for such equations involving fractional differential operators.

\section{BIBLIOGRAPHY}

1. I. Podlubniy. Fractional differential equations. Academic Press, New York (1999).

2. S.G. Samko, A.A. Kilbas, O.I. Marichev. Fractional integrals and derivatives: theory and applications. Gordon and Breach, Longhorne (1993).

3. T.D. Dzhuraev, A. Sopuev, M. Mamazhanov. Boundary value problems for equations of parabolichyperbolic type. FAN, Tashkent (1986).

4. B. Islomov, U. Baltaeva. Boudanry-value problems for a third-order loaded parabolic-hyperbolic type equation with variable coefficients // Electron. J. Differ. Equat. 2015, id 221 (2015).

5. U. Baltaeva, P. Agarwal. Boundary value problems for a third-order loaded equation with noncharacteristic type change boundaries // Math. Meth. Appl. Sci. 41:9, 3305-3315 (2018).

6. O.Kh. Abdullaev, K. Sadarangani. Non-local problems with integral gluing condition for loaded mixed type equations involving the Caputo fractional derivative// Electron. J. Differ. Equat. 2016, id 164 (2016).

7. K. Sadarangani, O.Kh. Abdullaev. About a problem for loaded parabolic-hyperbolic type equation with fractional derivatives // Int. J. Diff. Equat. 2016, id 9815796 (2016).

8. O.Kh. Abdullayev. Solvability of a non-local problem with integral gluing condition for mixed type equation with Erdelyi-Kober operators // Fract. Differ. Calc. 7:2, 371-383 (2017).

9. A.M. Nakhushev. A nonlocal problem and the Goursat problem for a loaded equation of hyperbolic type, and their applications to the prediction of ground moisture // Dokl. Akad. Nauk SSSR. 242:5. 1243-1247 (1978). [Sov. Math. Dokl. 19, 1243-1247 (1978).]

10. A.M. Nakhushev. Loaded equations and their applications. Nauka, Moscow (2012). (in Russian).

11. E.N. Zhuravleva, E.A. Karabut. Loaded complex equations in the problem of impact of jets // Comput. Math. Math. Phys. 51:5, 876-894 (2011).

12. I.S. Lomov. A theorem on unconditional basis property of roots vectors of second order weighted differential operators // Diff. Equat. 27:9, 1550-1563 (1991).

13. F. Bloom. Ill-posed problems for integro-differential equations in mechanics and electromagnetic theory. SIAM, Philadelphia (1981). 
14. K. Schumacher. Traveling-front solutions for integro-differential equations. II // In. "Biological growth and spread. Lecture notes in biomathematics", eds. W. Joger, H. Rost, P. Tautu. 38. 296-309 (1980).

15. N. Apreutesei, A. Ducrot, V. Volpert. Travelling waves for integro-differential equations in population dynamics // Discr. Cont. Dynam. Syst. B 11:3, 541-561 (2009).

16. A.V. Pskhu. Solution of boundary value problems fractional diffusion equation by the Green function method // Diff. Equat. 39:10, 1509-1513 (2003).

17. O.Kh. Abdullaev, A.A. Matchanova. Non-local boundary value problems for a loaded parabolichyperbolic type equation of third order involving Caputo operator // Bull. Inst. Math. 2018:5, 36-42 (2018).

Bozor Islomovich Islomov,

National University of Uzbekistan

named after Mirzo Ulugbek,

Universitetskaya str. 4,

100174, Tashkent, Uzbekistan

E-mail: islomovbozor@yandex.com

Obidjon Khayrullaevich Abdullaev,

V.I. Romanovsky Institute of Mathematics,

Universitetskaya str. 4-a,

100174, Tashkent, Uzbekistan

E-mail: obidjon.mth@gmail.com 